Kristin C. C. Petri*, Paula M. Hale, Paul L. Hofman and Lisbeth V. Jacobsen

\title{
Liraglutide pharmacokinetics and exposure- response in pediatric patients with type 2 diabetes
}

https://doi.org/10.1515/jpem-2020-0053

Received February 12, 2020; accepted June 30, 2020; published online August 18, 2020

\section{Abstract}

Objectives: Based on the ellipse trial, liraglutide was recently approved for use in pediatric patients with type 2 diabetes. We report the comparative exposure of liraglutide in pediatric vs. adult patients.

Methods: In this pharmacokinetic (PK) and exposureresponse meta-analysis, data from two pediatric trials (including ellipse) and two adult trials of liraglutide were compiled (1,137 PK observations from 116 patients) to determine the impact of body weight, age and sex on liraglutide exposure. The exposure-response relationship for glycated hemoglobin $\left(\mathrm{HbA}_{1 \mathrm{c}}\right)$ and body weight was compared between pediatric and adult patients. Additionally, the relationships between exposure and change from baseline in body mass index (BMI) and BMI standard deviation score (SDS) were assessed.

Results: The same liraglutide dose showed comparable exposure levels in pediatric and adult patients. Body weight and sex were the most important covariates for liraglutide exposure. There was an increasing response with higher liraglutide concentrations, and greater reductions were observed from baseline in $\mathrm{HbA}_{1 \mathrm{c}}$ at 26 weeks vs. placebo. A trend toward lower body weight, BMI and BMI SDS was observed at 26 weeks.

Conclusions: These results support use of the same liraglutide dosing regimen in children and adolescents, aged $\geq 10$ years, as that used in adults.

Keywords: exposure-response; liraglutide; pharmacokinetics; pediatric; type 2 diabetes.

\footnotetext{
*Corresponding author: Kristin C. C. Petri, Novo Nordisk A/S, Vandtårnsvej 108-110, DK-2860, Søborg, Denmark, Phone: +45 4444 8888, E-mail: kcc@novonordisk.com

Paula M. Hale, Novo Nordisk Inc., Plainsboro, NJ, USA

Paul L. Hofman, Liggins Institute, University of Auckland, Auckland, New Zealand

Lisbeth V. Jacobsen, Novo Nordisk A/S, Søborg, Denmark
}

\section{Introduction}

The prevalence of type 2 diabetes (T2D) in children and adolescents is increasing, and until recently, the only approved treatments in this population were insulin and metformin. The glucagon-like peptide- 1 analog liraglutide was recently approved for pediatric patients with T2D aged $\geqq 10$ years, based on the phase 3 randomized, placebocontrolled ellipse trial (NCT01541215) [1] results, which demonstrated that liraglutide improved glycemic control (both glycated hemoglobin $\left[\mathrm{HbA}_{1 \mathrm{c}}\right]$ and fasting plasma glucose) in pediatric patients with T2D [2]. Thus, based on improvement in glycemic control, liraglutide may help address the unmet need for treatment options in pediatric patients with T2D.

In adults, the liraglutide pharmacokinetics display dose-exposure proportionality, with body weight and sex being the most important covariates for liraglutide exposure [3]. A previous pharmacokinetic (PK) analysis of liraglutide comparing data from a pediatric T2D trial to data from two clinical pharmacology adult T2D trials showed that the clinically effective liraglutide dose in adults achieved the same range of exposure in pediatric patients aged 10 to <17 years [4]. Consequently, the adult dose was used in ellipse, and the population PK and exposure-response analysis for liraglutide (dosed to a maximum of $1.8 \mathrm{mg}$ once daily) are reported here.

Data from the previous combined PK analysis of liraglutide were analyzed together with the ellipse PK data set [4]. The historical data are from the following prespecified, randomized, liraglutide trials: Trial 1 (NCT00943501), double-blind trial assessing safety/ tolerability and PK in pediatrics; Trial 2 (NCT00993304), double-blind PK adult trial; Trial 3 (NCT00873223), PK open-label adult trial (see Supplementary material for trial designs). The adult trials were chosen based on relevant populations and availability of rich PK data to support the population PK model. The meta-analysis undertaken allowed a covariate analysis including the age-group (pediatric/adult).

Data from LEAD-3 (NCT00294723) were used for external validation, allowing comparison of ellipse with a larger phase 3 trial of adults with T2D [5]. 


\section{Materials and methods}

A first-order conditional estimation method was used to develop a base population PK model drawing on prior knowledge. A full model approach was used [6], and the final covariate model included effects of sex, age group (pediatric/adult) and body weight on apparent clearance (CL/F) and apparent volume of distribution (V/F). Observations below the lower limit of quantification (LLOQ) were excluded. The reference patient profile was an adult female (body weight: $90 \mathrm{~kg}$ ). For model qualification and possible limitations of the PK model, see Supplementary material.

The final model was used to estimate $\mathrm{CL} / \mathrm{F}$, area under the plasma concentration-time curve from time $0-24 \mathrm{~h}\left(\mathrm{AUC}_{0-24}\right)$ and average concentrations $\left(\mathrm{C}_{\mathrm{avg}}\right)$ at the steady state in the pediatric population. Simulations of liraglutide concentrations included between-patient variability for pediatric and adult patients of the same body weight. Original $\mathrm{C}_{\mathrm{avg}}$ estimates were used for LEAD-3, including adults randomized to 1.2 or $1.8 \mathrm{mg}$ of liraglutide [3].

An exposure-response analysis for $\mathrm{HbA}_{1 \mathrm{c}}$, with baseline $\mathrm{HbA}_{1 \mathrm{c}}$, body weight and sex as covariates, was conducted for liraglutide. The analysis comprised exposure data regarding individual $\mathrm{C}_{\mathrm{avg}}$ values obtained from the population $\mathrm{PK}$ analysis and response $\left(\mathrm{HbA}_{1 \mathrm{c}}\right.$ change from baseline to week 26). $C_{\text {avg }}$ was set to $\mathrm{LLOQ} / 2(0.015 \mathrm{nmol} / \mathrm{L})$ for liraglutide-treated patients with samples below the LLOQ at week 26 . $C_{\text {avg }}$ was set to zero for placebo-treated patients. Patients on active treatment were divided into quantiles based on their $\mathrm{C}_{\mathrm{avg}}$. For each exposure quantile and placebo group, the mean ( $95 \%$ confidence interval [CI]) response was plotted against median liraglutide concentration.

NONMEM, version 7.3.0, was used for the population PK analysis. $R$, version 3.2.3, was used for compiling data sets, plotting data and exposure-response analysis.

The trial was approved by local institutional review boards, and all patients (or their legally authorized representative) provided written informed consent; the trial was conducted according to the Declaration of Helsinki.

\section{Results}

\section{PK data sets}

The PK data set comprised data from ellipse and the three historical trials and contained 1,137 PK observations from
116 patients with T2D (Supplementary Table 1). The ellipse data set comprised $251 \mathrm{PK}$ observations from 59 patients. In total, 326 PK observations were recorded from Trial 1, 144 were recorded from Trial 2 and 416 were recorded from Trial 3. Overall, baseline characteristics and demographics were broadly comparable across all trials. Differences included greater racial diversity in ellipse vs. the other trials and different mean baseline body weights, varying from the lowest in Trial $2(82.4 \mathrm{~kg})$ to the highest in Trial $1(114.7 \mathrm{~kg})$, with overlap between the body weight ranges of the trials (Supplementary Table 2).

\section{Evaluation of covariate effects on liraglutide exposure}

Body weight was the most important covariate for liraglutide exposure (Figure 1), with increasing body weight associated with lower exposure $\left(\mathrm{C}_{\mathrm{avg}}\right)$ (Supplementary Figure 1). Relative exposure was lower in males than in females (ratio: 0.72 [95\% CI: 0.62; 0.85]). Age was included as a categorical covariate (adult vs. pediatric), but the effect on exposure was not found to be clinically relevant.

\section{Liraglutide exposure}

Simulation with the final model showed overlapping concentration-time profiles for an adult reference patient and a comparable pediatric patient (both females with a baseline body weight of $90 \mathrm{~kg}$ ). The $\mathrm{C}_{\mathrm{avg}}$ for liraglutide overlapped substantially between pediatric and adult patients (Figure 2). External validation was provided by observing that $\mathrm{C}_{\text {avg }}$ for liraglutide overlapped substantially between pediatric and adult patients for all liraglutide doses in ellipse and LEAD-3 (Figure 3). Both the individual $\mathrm{CL} / \mathrm{F}$ and exposure estimates $\left(\mathrm{C}_{\mathrm{avg}}\right.$ and $\left.\mathrm{AUC}_{0-24}\right)$ for

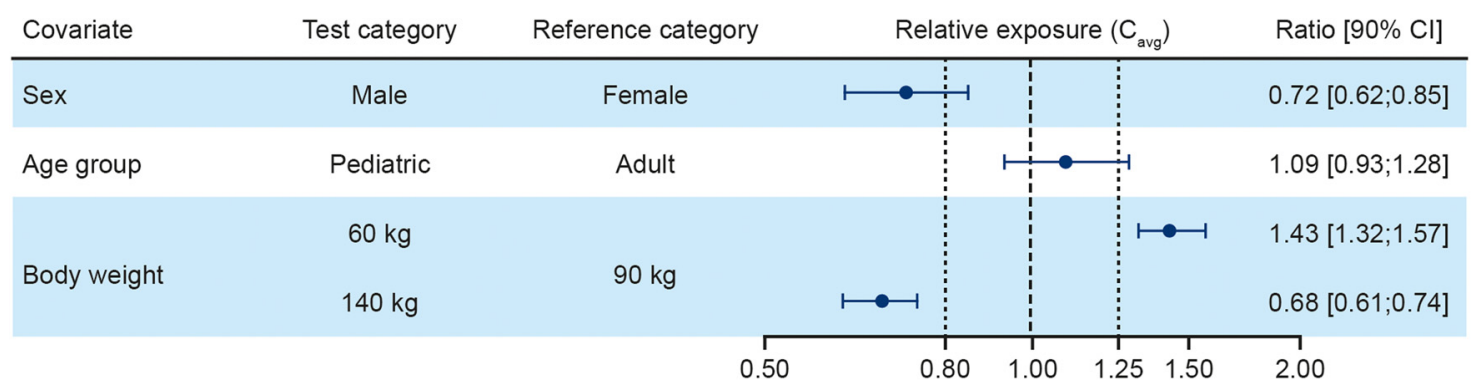

Figure 1: Forest plot of covariate analysis for liraglutide exposure expressed as steady-state, dose-normalized, and mean liraglutide concentrations relative to a reference patient. Body weight test categories ( 60 and $140 \mathrm{~kg}$ ) represent the $5 \%$ and $95 \%$ percentiles, respectively. Vertical lines indicate the acceptance interval for bioequivalence $(0.80 ; 1.25)$ and relative exposure in the reference patient set to 1 . Data from ellipse and Trials 1, 2 and 3. $\mathrm{C}_{\text {avg }}$, steady-state average liraglutide concentration; $\mathrm{Cl}$, confidence interval. 
liraglutide were comparable between pediatric and adult patients (Supplementary Table 3).

\section{Results from the exposure-response analysis}

Higher liraglutide exposure was associated with greater $\mathrm{HbA}_{1 \mathrm{c}}$ reductions (Figure 4). The exposure-response relationships for body weight, body mass index (BMI) and BMI standard deviation score (SDS) did not differ significantly from baseline to 26 weeks in pediatric patients in ellipse (Supplementary Figure 2).

\section{Discussion}

Overall, the results from this population PK and exposureresponse meta-analysis are comparable to previous results from pediatric and adult clinical trials of liraglutide $[4,6,7]$.

Comparable exposure levels of liraglutide were observed between pediatric and adult patients. Consistent with previous reports, body weight was the most important covariate for exposure $[4,6,7]$. The lower exposure in males has also previously been found in adults $[4,6,7]$. Body weight ranges for pediatric patients in ellipse were

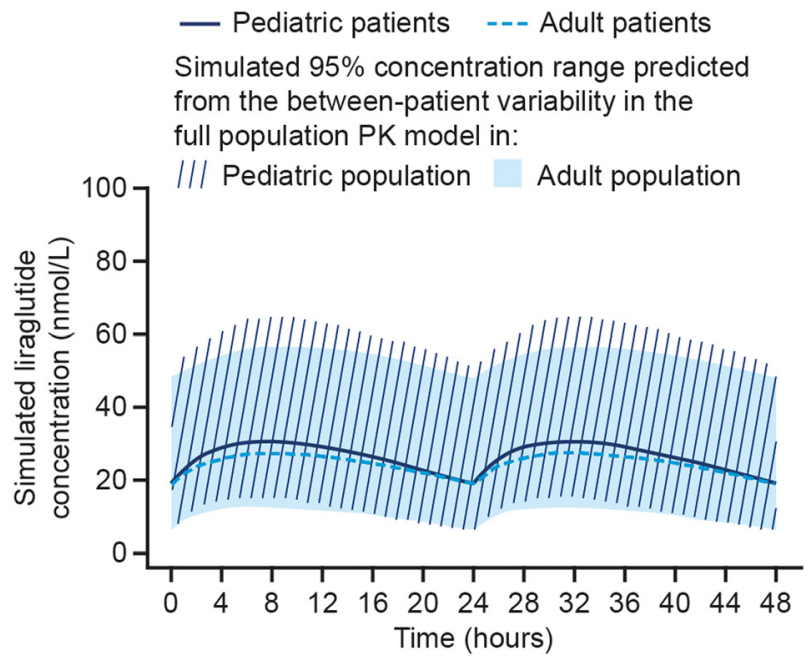

Figure 2: Simulated steady-state concentration-time profiles following $1.8 \mathrm{mg}$ of liraglutide once daily for pediatric and adult patients. Simulation of liraglutide concentrations from a full model including between-patient variability. Lines are model-derived mean population profiles vs. time, covering two dosing intervals for two patient profiles (pediatric and adult female patients, body weight: $90 \mathrm{~kg} ; \mathrm{n}=1,000$ replications in each group). PK, pharmacokinetic.

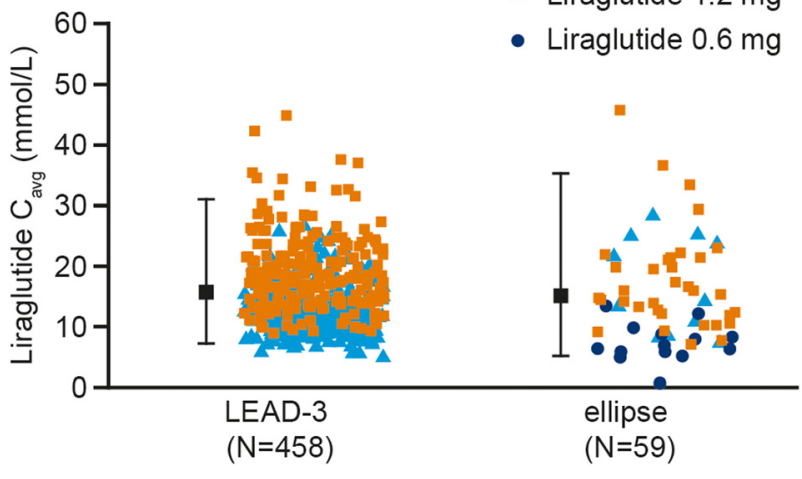

Figure 3: Individual average steady-state concentrations in the adult LEAD-3 and pediatric ellipse trials including patients on all doses. Data (unadjusted for body weight) are individual modelderived $C_{\text {avg }}$ (symbols) and mean ( $90 \%$ range [5th-95th \%o]). $C_{\text {avg }}$ estimates from a population PK analysis of LEAD-3 and from the full model for the ellipse trial. LEAD-3: adults randomized to 1.2 or $1.8 \mathrm{mg}$ of liraglutide; ellipse: highest achieved maintenance dose $(0.6,1.2$ or $1.8 \mathrm{mg})$. Mean body weight was 93.1 (LEAD-3) and $94.7 \mathrm{~kg}$ (ellipse), respectively. $C_{\text {avg }}$, steady-state average concentration; $n$, number of patients; PK, pharmacokinetic.

similar to those in adults in LEAD-3 and in line with results reported across several liraglutide adult phase 3 trials $[4,8]$.

$\square$ Patients randomized to liraglutide

Patients randomized to placebo

Median and $90 \%$ exposure ranges for patients grouped by highest achieved dose:

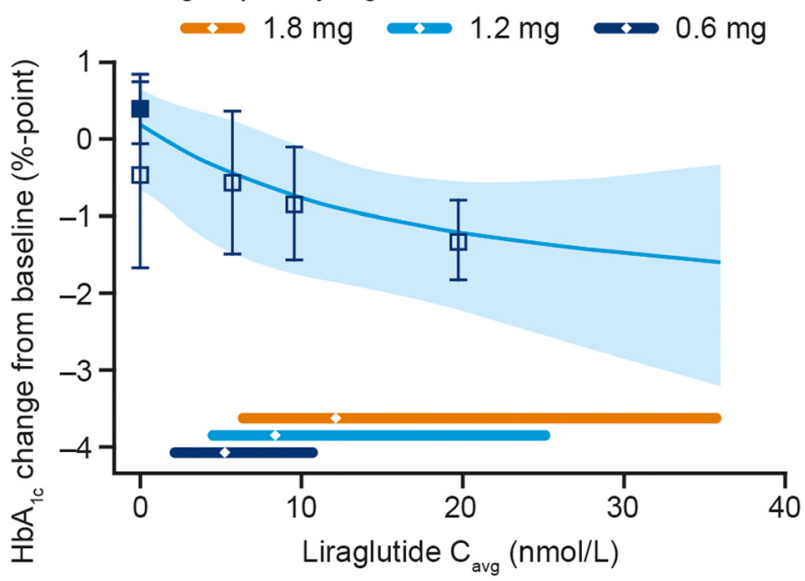

Figure 4: $\mathrm{HbA}_{1 \mathrm{c}}$ change from baseline vs. liraglutide exposure in pediatric patients. The line through the data represents the covariate-adjusted model-derived exposure-response relationship with $90 \% \mathrm{Cl}$. Estimates for ellipse based on data from week 26, $\mathrm{n}=109 . \mathrm{C}_{\text {avg }}$ set to $\mathrm{LLOQ} / 2(0.015 \mathrm{nmol} / \mathrm{L})$ for liraglutide-treated patients with sample below the LLOQ at week 26 , and $C_{\text {avg }}$ to 0 $\mathrm{nmol} / \mathrm{L}$ for placebo-treated patients. $C_{\text {avg }}$, steady-state average concentration; $\mathrm{Cl}$, confidence interval; $\mathrm{HbA}_{1 \mathrm{c}}$, glycated hemoglobin; $\mathrm{n}$, number of patients; LLOQ, lower limit of quantification. 
In the exposure-response analysis, a higher liraglutide exposure was associated with greater $\mathrm{HbA}_{1 \mathrm{c}}$ reductions at 26 weeks. A trend toward lower body weight, BMI and BMI SDS at 26 weeks was also observed with increasing liraglutide exposure. Change from baseline in body weight at week 26 was not significantly different between patients receiving liraglutide or placebo in ellipse, although the mean difference in weight loss between the treatment groups was similar to that observed in adult trials [2].

This analysis confirms previous findings, wherein the population PK model has been shown to describe PK profiles well $[4,6]$, and supports that the adult liraglutide doses (up to $1.8 \mathrm{mg}$ ) are appropriate for pediatric patients aged $\geq 10$ years.

\section{Conclusions}

Similar liraglutide exposure-responses were observed in the pediatric ellipse population and adult patients. Based on the overlapping body weight ranges expected for pediatric and adult patients with T2D, and similar liraglutide exposure, our results support use of the same liraglutide doses in children/adolescents as in adults. The same starting dose as used in adults can be escalated to improve glycemic control, based on individual responses in pediatric patients, up to $1.8 \mathrm{mg}$ or the maximally tolerated dose. The recently approved indication of liraglutide in pediatrics (age 10-17 years and T2D) provides an additional treatment option for this population.

Acknowledgments: Medical writing and editorial support were provided by Aneela Majid, $\mathrm{PhD}$, from Watermeadow Medical, an Ashfield company, part of UDG Healthcare plc, funded by Novo Nordisk.

Research funding: The ellipse trial and this analysis were sponsored by Novo Nordisk. The funder was involved in the study design, collection, analysis, and interpretation of data, writing of the report, and decision to submit the report for publication.

Author contributions: All the authors have accepted responsibility for the entire content of this submitted manuscript and approved submission.
Competing interests: $\mathrm{PH}$ reports consultation fees from Sandoz and Becton Dickenson. KCC, LVJ and PMH are Novo Nordisk full-time employees and shareholders.

Ethical approval: The trial was approved by local institutional review boards and all patients (or their legally authorized representative) provided written informed consent and was conducted according to the Declaration of Helsinki.

\section{References}

1. US Food and Drug Administration. FDA approves new treatment for pediatric patients with type 2 diabetes; 2019. Available from: https://www.fda.gov/news-events/press-announcements/fdaapproves-new-treatment-pediatric-patients-type-2-diabetes [Accessed 27 June 2019].

2. Tamborlane WV, Barrientos-Perez M, Fainberg U, Frimer-Larsen $H$, Hafez M, Hale PM, et al. Liraglutide in children and adolescents with type 2 diabetes. N Engl J Med 2019; 381: 637-46.

3. Ingwersen SH, Khurana M, Madabushi R, Watson E, Jonker DM, Le Thi TD, et al. Dosing rationale for liraglutide in type 2 diabetes mellitus: a pharmacometric assessment. J Clin Pharmacol 2012; 52: 1815-23.

4. Petri KC, Jacobsen LV, Klein DJ. Comparable liraglutide pharmacokinetics in pediatric and adult populations with type 2 diabetes: a population pharmacokinetic analysis. Clin Pharmacokinet 2015; 54: 663-70.

5. Garber A, Henry R, Ratner R, Garcia-Hernandez PA, RodriguezPattzi H, Olvera-Alvarez I, et al. Liraglutide vs. glimepiride monotherapy for type 2 diabetes (LEAD-3 Mono): a randomised, 52-week, phase III, double-blind, parallel-treatment trial. Lancet 2009; 373: 473-81.

6. Ingwersen SH, Petri KC, Tandon N, Yoon KH, Chen L, Vora J, et al. Liraglutide pharmacokinetics and dose-exposure response in Asian subjects with type 2 diabetes from China, India and South Korea. Diabetes Res Clin Pract 2015; 108: 113-19.

7. Jacobsen LV, Flint A, Olsen AK, Ingwersen SH. Liraglutide in type 2 diabetes mellitus: clinical pharmacokinetics and pharmacodynamics. Clin Pharmacokinet 2016; 55: 657-72.

8. Niswender K, Pi-Sunyer X, Buse J, Jensen KH, Toft AD, Russell-Jones $D$, et al. Weight change with liraglutide and comparator therapies: an analysis of seven phase 3 trials from the liraglutide diabetes development programme. Diabetes Obes Metabol 2013;15: 42-54.

Supplementary Material: The online version of this article offers supplementary material https://doi.org/10.1515/jpem-2020-0053 